\title{
Introduction to the focused section on intelligent robotics for civil infrastructure
}

\author{
Yang Wang ${ }^{1}$ Yunhua $\mathrm{Li}^{2} \cdot$ Thomas Bock ${ }^{3} \cdot$ Jerome P. Lynch $^{4} \cdot$ Jouni Mattila $^{5}$
}

Published online: 25 July 2017

(C) Springer Nature Singapore Pte Ltd. 2017

\section{Introduction}

Intelligent robots represent the sophisticated incorporation of robotics, cyber physical systems and artificial intelligence. A great variety of intelligent robots are finding exciting applications and playing important roles in civil infrastructure systems. Taking the field of structural health monitoring for example, researchers have investigated the concept of mobile sensors (Akyildiz et al. 2002; LaMarca et al. 2002), where robots can autonomously or semi-autonomously move sensors around a structure and conveniently change measurement locations. In particular, smallsize crawling and flying robots have been investigated for structural monitoring and inspection (Hirose et al. 2015; Guo et al. 2012; Zhu et al. 2010; Zhou et al. 2016). Meanwhile, intelligent robotics and machinery of much larger size are also playing an important role in

Yang Wang

yang.wang@ce.gatech.edu

Yunhua Li

yhli@buaa.edu.cn

Thomas Bock

thomas.bock@br2.ar.tum.de

Jerome P. Lynch

jerlynch@umich.edu

Jouni Mattila

jouni.mattila@tut.fi

Georgia Institute of Technology, Atlanta, USA

2 Beihang University, Beijing, People's Republic of China

3 Technical University of Munich, Munich, Germany

4 University of Michigan, Ann Arbor, USA

5 Tampere University of Technology/IHA, Tampere, Finland construction and maintenance of civil infrastructure. Furthermore, the combination of robotics, electronics, network systems, pattern recognition, and intelligent computing has also thrust a significant amount of work in smart structural technologies (Li et al. 2013). Containing articles in both theoretical and practical/experimental development, this focused section aims to disseminate current advances of various robotics technologies for civil infrastructures.

\section{Articles in this focused section}

The article "Robotic Sensing and Object Recognition from Thermal-Mapped Point Clouds" presents an innovative method for robotic sensing and object recognition from thermal-mapped point clouds, using thermal data from infrared camera to fuse 3D point clouds. A novel robotic hybrid system is developed. The multimodal data is combined with a data fusion process based on texture mapping. The automatic object recognition is performed by two cases including segmentation with thermal data and classification with scanned geometric features. The research results show that the proposed method is validated with the scan data collected from an entire building floor, and the thermal integrated object recognition approach can achieve better performance than a geometry only-based approach for objects in the tested environment including humans, display monitors and light fixtures.

The article "Wall-Climbing Robot for Non-Destructive Evaluation Using Impact-Echo and Metric Learning SVM" studies the application of the Impact-Echo (IE) acoustic inspection method in non-destructive evaluation and detection of the concrete structure. The authors present a novel climbing robot, namely Rise-Rover, to perform automated IE signal collection from concrete structures and 
IE signal analyzing based on machine learning techniques. Rise-Rover is an inspection robot with a novel and enhanced absorption system to support heavy load, and crawler-like suction cups to maintain high mobility performance while crossing small grooves. Various signal processing methods, including Fourier transform and wavelet transform for feature detection from collected IE signals, are investigated, and a distance metric learning based support vector machine (SVM) approach is newly proposed to automatically classify the IE signals. Field tests on a concrete bridge deck have demonstrated the efficiency of the proposed robot system in automatic health condition assessment for concrete structures.

The article "RABIT: Implementation, Performance Validation and Integration with Other Robotic Platforms for Improved Management of Bridge Decks" presents a highly integrated large robotic system for concrete bridge deck inspection. Traditional inspection is mainly performed by human inspectors that operate individual hardware platforms. In comparison, besides vision sensing, the RABIT system integrates a multitude of platforms into one autonomous system, including electrical resistivity, ground-penetrating radar, impact echo and ultrasonic surface wave method. Such integration significantly increases the productivity and data resolution for bridge deck inspection, making it possible to simultaneously capture a plurality of concrete deterioration processes that may take place in the field. This unprecedented incorporation of a multitude of large sensor arrays offers exciting new opportunity for the condition monitoring of transportation infrastructure.

The article "Reconfigurable Swarm Robotic Systems for Structural Health Monitoring: A Brief Review" surveys the features, typical applications and present statuses of the development of reconfigurable swarm robots (RSR). This authors have summarized the main advantages of RSR: being modular, on-site reconfigurable, multifunctional, incrementally assemble-able, reusable, fault-tolerant, and even repairable on the orbit. The review article argues that RSR offer great potential and advantages from the perspective of monitoring and assessment of civil and mechanical systems. Moreover, a roadmap for future research has also been outlined based on the limitations of the current methods and anticipated needs of future inspection systems.

The article by Goorts et al., entitled "Structural Control using a Deployable Autonomous Control System", is another demonstration of novel robotics applications in civil structures. As noted by the authors, this deployable autonomous control system is particularly suitable for situations in need of a temporary control setup. While conventional control devices, either passive, semi-active, or active, are often permanently installed at fixed locations on a structure, this article proposes to use an Unmanned Ground Vehicle (UGV) as a mobile control devices for reducing structural vibration. Equipped with an electromechanical mass damper, the UGV can automatically travel around and choose an optimal location for applying damping force to the structure. With vision sensors onboard, the UGV simultaneously locates and maps the structural environment it travels in. A movable/deployable control device particularly provides the advantage of multimodal control by optimally choosing its desired location. Another apparent advantage is less dependence on an accurate structural dynamics model, which often can be difficult to obtain for large civil structures. The innovative concept has been successfully validated using a $16.7 \mathrm{~m}$ long pedestrian bridge.

\section{Summary and conclusion}

We believe that intelligent robots will continue to generate exciting and impactful applications on civil infrastructures. These may include high-efficiency operation of large infrastructures, mobile sensor network and robotic inspection for civil structures, smart materials and intelligent structures, robotic rehabilitation of large civil structures, and novel actuation and control techniques for construction machinery and construction automation, among others.

Finally, the guest editors would like to acknowledge the great efforts by the authors and anonymous reviewers towards this focused section. We would also like to extend our gratitude to the outstanding journal staff at IJIRA that made this focused section possible.

\section{References}

Akyildiz, I.F., Su, W., Sankarasubramaniam, Y., Cayirci, E.: A survey on sensor networks. Commun Mag IEEE 40, 102-114 (2002)

LaMarca, A., Brunette, W., Koizumi, D., Lease, M., Sigurdsson, S.B., Sikorski, K., Fox, D., Borriello, G.: Making sensor networks practical with robots. In: Proceedings of the first international conference on pervasive computing, Zurich, Switzerland, 2002

Hirose, M., Xiao, Y., Zuo, Z., Kamat, V.R., Zekkos, D., Lynch, J.: Implementation of UAV localization methods for a mobile postearthquake monitoring system. In: Proceedings of the 2015 IEEE workshop on environmental, energy, and structural monitoring systems (EESMS), 2015

Guo, J., Lee, K.M., Zhu, D., Yi, X., Wang, Y.: Large-deformation analysis and experimental validation of a flexure-based mobile sensor node. Mechatron. IEEE/ASME Trans. 17, 606-616 (2012)

Zhu, D., Yi, X., Wang, Y., Lee, K.-M., Guo, J.: A mobile sensing system for structural health monitoring: design and validation. Smart Mater. Struct. 19, 055011 (2010)

Zhou, H., Hirose, M., Greenwood, W., Xiao, Y., Lynch, J., Zekkos, D., Kamat, V.: Demonstration of UAV deployment and control 
of mobile wireless sensing networks for modal analysis of structures. In: Proceedings of SPIE, sensors and smart structures technologies for civil, mechanical, and aerospace systems, San Diego, CA, USA, 2016

Li, Y., Wang, Y., Chase, J.G., Mattila, J., Myung, H., Sawodny, O.: Survey and introduction to the focused section on mechatronics for sustainable and resilient civil infrastructure. IEEE/ASME Trans. Mechatron. 18, 1637-1646 (2013)

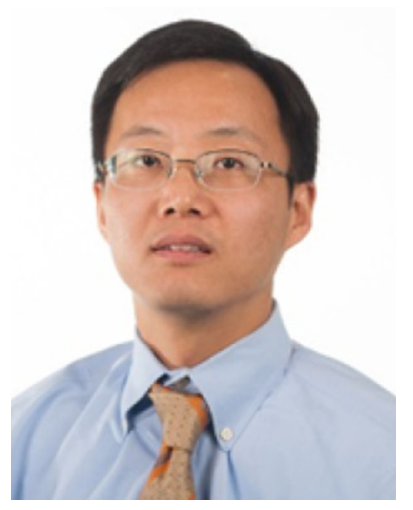

Yang Wang is an Associate Professor in the School of Civil and Environmental Engineering at the Georgia Institute of Technology. After completing a B.S. and an M.S. degree in Civil Engineering at Tsinghua University in Beijing, China, he received a Ph.D. degree in Civil Engineering at Stanford University in 2007, as well as an M.S. degree in Electrical Engineering. Dr. Wang's research interests include structural health monitoring and damage detection, decentralized structural control, wireless and mobile sensors, and structural dynamics. He received an NSF Early Faculty Career Development (CAREER) Award in 2012 and a Young Investigator Award from the Air Force Office of Scientific Research (AFOSR) in 2013. Since 2011, Dr. Wang has served as an Associate Editor for the ASCE (American Society of Civil Engineers) Journal of Bridge Engineering.

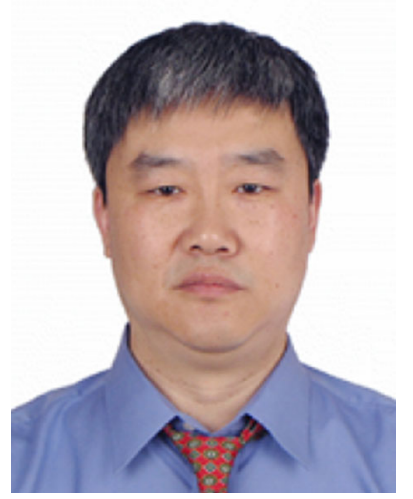

Yunhua Li received the M.S. degree in mechanical engineering from Jilin University of Technology, Changchun, China, in 1988, and $\mathrm{Ph}$. D degree in Mechatronics from Xi'an Jiaotong University, Xi'an, China, in 1994, respectively. Dr Li was employed as a Postdoctoral research fellow in Beihang University, China, and National Chengkung University, Taiwan, from December 1994 to October 1996 and from July 1997 to August 1997, respectively. Since 1999, Dr. Li has been a Full Professor in Mechanical Engineering in Beihang University. His research interests include construction machinery and service robot, hydraulic and mechatronic servo control, and power transmission and control of aircraft, mobile robots and engineering vehicle. Dr Li has won more than ten Scientific and Technological Awards in China. He has published more than 260 refereed journal and conference papers, and four books. During 2010-2015, Dr. Li was a technical editor of IEEE/ASME Transactions on Mechatronics.

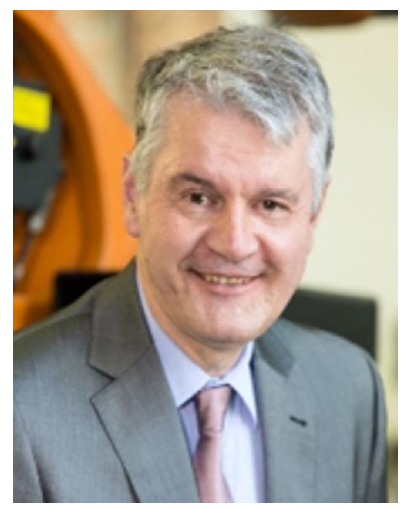

Thomas Bock is a professor of building realization and robotics at Technische Universität München (TUM). He got his education in architecture at Stuttgart University in Germany, in engineering at IIT in Chicago in USA as Fulbright Scholar and in construction automation and robotics at University of Tokyo in Japan. $\mathrm{He}$ spent 10 years abroad mostly in EU, USA and Japan. He holds an US FAA commercial pilot license with IFR and MEL ratings. Professionally he worked in the construction industry in Germany, USA, Iran and Japan, designed and built in Germany, Spain and Costa Rica. Academically he focuses since 35 years on automation and robotics in building construction, from the planning, prefabrication, on site production and utilization phases to the reorganization and deconstruction of a building. He started the national French construction robotics initiative in 1989 as a researcher for CNRS and in 1992 the first EU ESPRIT project on mobile construction robot. Overall he was involved in the development of 50+ robotic systems for stationary production of modules made in concrete-masonry-timber-steel, on site robotic systems, automated construction and deconstruction sites and mechatronic building subsystems for daily life/work support. He is a member of several boards of directors of international associations and is a member of several international academies in Europe, Americas and Asia. He consulted several international ministries and evaluates research projects for various international funding institutions. He holds fellowship, honorary doctor and professorship degrees and visiting professorships. Professor Bock serves on several editorial boards and since 2017 is associate editor of the Springer journal Construction Robotics. He heads various working commission and groups of international research organizations, authored and co-authored about 450 articles and since 2015 the world wide first Construction Robotics handbook series consisting of five volumes at Cambridge University Press.

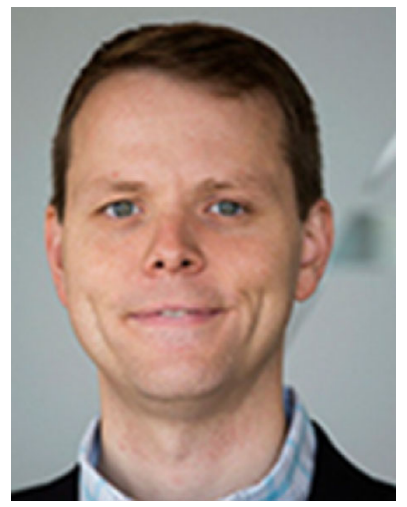

Jerome $\mathbf{P}$. Lynch received the B.E. degree in civil and environmental engineering from the Cooper Union, New York, NY, USA, in 2008 and the M.S. and $\mathrm{Ph} . \mathrm{D}$. degrees in civil and environmental engineering and the second M.S. degree in electrical engineering from Stanford University, Stanford, CA, USA, in 1998, 2002, and 2003, respectively. $\mathrm{He}$ is a Professor of Civil and Environmental Engineering at the University of Michigan, Ann Arbor, MI, USA. He is also a Professor of Electrical Engineering and Computer Science by courtesy. His current research interests are in the areas of wireless cyber-physical systems, cyberinfrastructure tools for management of structural monitoring data sets, and nanoengineered thinfilm sensors for damage detection and structural health monitoring. Dr. Lynch has been awarded the 2005 Office of Naval Research (ONR) Young Investigator Award, the 2009 National Science Foundation (NSF) CAREER Award, the 2009 Presidential Early Career Award for Scientists and Engineers (PECASE), the 2012 
ASCE EMI Leonardo da Vinci Award, and the 2014 ASCE Huber Award.

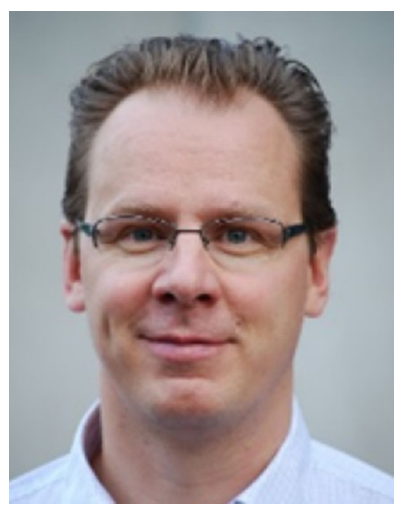

Jouni Mattila received the M.Sc. degree in 1995 and Dr. Tech. degree in 2000, both from the Tampere University of Technology (TUT), Tampere, Finland. He was a Visiting Researcher at the University of British Columbia, Vancouver, BC, Canada, in years 1998-2000. As a Senior Systems Engineering Consultant, he has been involved in numerous industrial research projects, including the Sandvik AutoMine with Hermia Group Ltd., since 1996. He is currently a Professor in Machine Automation in the
Department of Intelligent Hydraulics and Automation, TUT. Since the last 15 years, he has been a Program Manager on ITER fusion reactor maintenance projects involving research on heavy-duty hydraulic robotic manipulators. He has recently coordinated a Marie Curie ITN-project with 15 Ph.D. students across the EU to develop mobile service robotics for scientific infrastructures, such as CERN. He has authored or coauthored more than 100 papers published in international journals and conference proceedings. His research interests include machine automation, developing nonlinear modelbased control systems for robotic mobile manipulators and off-highway machinery, and energy-efficiency of fluid power and hybrid systems. Dr. Mattila is currently a Technical Editor of the IEEE/ ASME Transactions on Mechatronics. 